\title{
THE CORRELATIN BETWEEN MAJOR CRITERIA OF AHP FOR GOVERNMENT R\&D PROGRAM IN KOREA
}

\author{
Dong-Guen Kim \\ R\&D Feasibility Analysis Center \\ Korea Institute of S\&T Evaluation and Planning (KISTEP) \\ Seoul, South Korea \\ E-mail: dgkim@kistep.re.kr
}

\begin{abstract}
The preliminary feasibility study is carried for the newly proposed large-scaled government programs in Korea since 1998. PFS about research and development (R\&D) programs also became compulsory since 2008. In case of a PFS on R\&D programs, there are three major criteria about technology, policy and economic effects. Each expert evaluates the program about three major criteria and the score of each criterion is aggregated into overall score. The newly proposed program is finally evaluated into two alternatives, feasible or infeasible.

In this study, the correlation between three major criteria and overall score is analyzed. In addition, the feasible cases in which overall score are more than 0.5 and the unfeasible cases in which overall score are less than 0.5 are compared. The results show that the major criteria of preliminary feasibility study have correlation and the difference on feasible and infeasible programs is existed.
\end{abstract}

Keywords: preliminary feasibility study, major criteria, correlation.

\section{Introduction}

In Korea, the preliminary feasibility study is carried for the newly proposed large-scaled government programs, since 1999. At that time, the preliminary feasibility study is carried for only SOC programs such as railway and highway. However, as the budget for research and development programs has grown and these programs are undertaken for the medium and long-term period, the preliminary feasibility study about research and development $(\mathrm{R} \& \mathrm{D})$ programs also became compulsory since 2008 by National finance act.

In case of the preliminary feasibility study on government R\&D programs, three major criteria are applied to measure technology, policy, and economics aspects. Technological feasibility analysis consists of three sub-criteria such as R\&D logic analysis (appropriateness of the research plan), technological viability, and overlap possibility (uniqueness $\&$ relevance). The $\mathrm{R} \& \mathrm{D}$ logic analysis is about planning process, program goal, specified sub-plan, and organization system. Concrete and organized plans are highly evaluated and a method of program logic model which disassembles a program into input, activity, output, outcome, and impact is used to understand the concept of the program. Technological viability assessment is about national level in the technology field, technology level assessment, scientific literature or patent analysis, and quantitative 
or qualitative index. Viable plans are highly evaluated. The overlap possibility assessment is to examine whether the program is overlapping with previously launched R\&D program. The criteria to decide a similarity among programs are program's objectives, goals, implementation processes, and research area.

The feasibility analysis on policy consists of sub-criteria such as relevance to national plans \& priority, cooperation among organizations, risk factors. The consistency and initiative of program help to understand the position of examined programs in the whole governmental policy, and risk analysis can help to identify risk included in examined program.

Economic Analysis consists of sub-criteria such as appropriateness of the cost estimation and economic benefit or scientific/technical effectiveness. Appropriateness of the cost estimation is to examine the way to estimate the total cost of the program and the subprograms. Sometimes the appropriate cost of the program is analyzed using a comparison with similar case ones. Economic benefit or scientific/technical effectiveness is measured by net present value, benefit cost ratio, or benefit cost of cost effectiveness analysis.

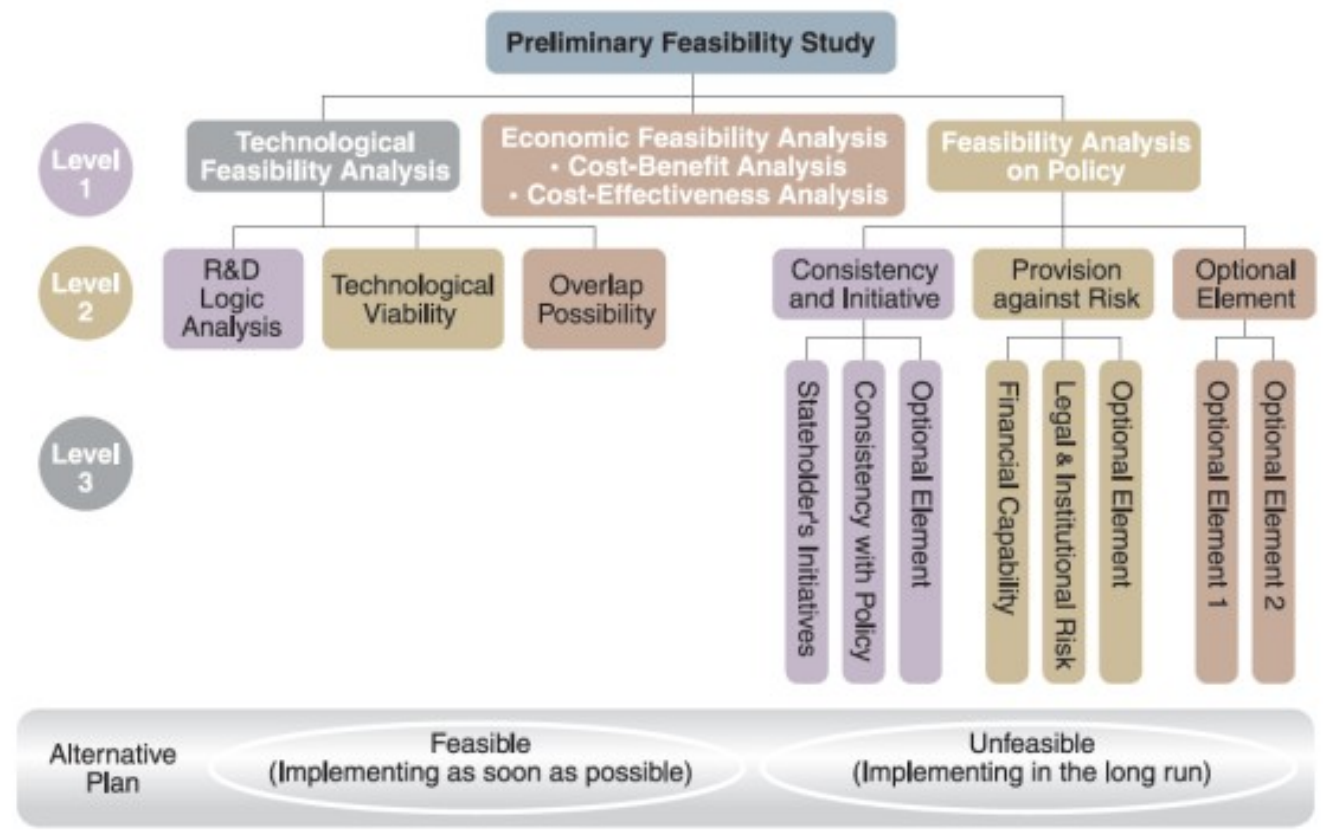

Usually, six experts evaluate one program and each expert evaluates the program about various sub-criteria of three major criteria. The score of each criterion is aggregated into overall score. In government guideline, there is the weight range for each major criterion. When the benefit-cost analysis is happen, the weight range of technological feasibility analysis is $30 \% \sim 40 \%$, and the weight range of feasibility analysis on policy is $20 \% \sim 30 \%$. The weight range of economic Analysis on policy is $40 \% \sim 50 \%$.

The newly proposed program is finally evaluated into two alternatives, feasible(implementing as soon as possible) or infeasible(implementing in the long run).

In this study, preliminary feasibility studies on government R\&D programs, which have been carried out in 2012, are analyzed. Three major criteria are examined in order to identify the possible correlations, such as similarity, cooperative relations, auxiliary 
relations, rivalry relations, competitive relations, and substitute relations. Also, the feasible cases in which overall score are more than 0.5 and the unfeasible cases in which overall score are less than 0.5 are compared.

\section{Correlation between major criteria}

Various methods can be used to assess correlation between major criteria in the preliminary feasibility studies on government $R \& D$ programs. In this study, correlation in scoring is analyzed about 100 evaluations of 17 government $R \& D$ programs. Correlation in scoring means that the relation between scores of two major criteria such as score of technological feasibility analysis and score of economic Analysis. The Pearson correlation coefficient is used to indicate the direction of relationship between major criteria.

The Pearson correlation coefficient is widely used to identify the relationship between elements. It is a measure of the correlation, which is linear dependence, between two variables $\mathrm{X}$ and $\mathrm{Y}$, giving a value between +1 and -1 inclusive. Usually, it is widely used as a measure of the strength of linear dependence between two variables. If the variables $\mathrm{X}$ and $\mathrm{Y}$ are identical, the correlation coefficient is +1 and 0 if different and -1 if the variables are identical in opposite direction.

In this study, the correlation coefficient was calculated on weighting factors applied to major criteria in decision making structures of the preliminary feasibility study. Major criteria with a certain significance level were considered; significance level $5.0 \%$ and $1.0 \%$.

Table 1. Results of Pearson correlation coefficient analysis

\begin{tabular}{|c|c|c|c|}
\hline & Technology & Policy & Economic \\
\hline Technology & 1 & $0.432^{* *}$ & $0.778^{* *}$ \\
\hline Policy & $0.432^{* *}$ & 1 & $0.203^{*}$ \\
\hline Economic & $0.778^{* *}$ & $0.203^{*}$ & 1 \\
\hline
\end{tabular}

The results of Pearson correlation coefficient analysis show that all major criteria have positive correlation with other major criteria. The correlation between score of technological feasibility analysis and score of economic analysis and the correlation between score of technological feasibility analysis and score of feasibility analysis on policy are strong positive satisfying significance level $1.0 \%$. The correlation between score of feasibility analysis on policy and score of economic analysis are positive satisfying significance level $5.0 \%$. In addition, the correlation between score of technological feasibility analysis and score of economic analysis is more positive than the correlation between score of technological feasibility analysis and score of economic analysis.

Such results imply that program evaluators tend to consider simultaneously technological aspect and economic aspect on each program. On the other hand, feasibility analysis on policy is evaluated, independently to other major criteria. Specially, the policy aspect of a new program tends to be independent to economic aspect of the program. It means that economic feasibility must be considered when new R\&D program is planned by governmental policy.

\section{Comparison on alternative plan selection}

$\begin{aligned} & \text { International Symposium of } \\ & \text { the Analytic Hierarchy } \\ & \text { Process }\end{aligned}$
June 29- July 2, 2014


In addition, the feasible cases in which overall scores are more than 0.5 and the unfeasible cases in which overall scores are less than 0.5 are compared. In 70 evaluations among 100 evaluations, overall scores are more than 0.5 and the program is evaluated finally to feasible cases. On the other hand, in 30 evaluations overall score are more than 0.5 and the program is evaluated finally to unfeasible cases.

Table 2. Comparison between feasible cases and unfeasible cases

\begin{tabular}{|c|c|c|c|c|}
\hline & \multicolumn{4}{|c|}{ The number of criteria in which score is more than 0.5} \\
\hline & Three criteria & Two criteria & One criterion & Zero criterion \\
\hline Feasible Cases & 47 & 21 & 2 & \\
\hline Unfeasible Cases & & & 16 & 14 \\
\hline
\end{tabular}

When the program is evaluated into feasible case, most evaluators $(67 \%)$ positively evaluated all major criteria. On the other hand, when the program is evaluated into unfeasible case, only half evaluators (47\%) negatively evaluated all major criteria.

\section{Key References}

Thomas L. Saaty, Luis G. Vargas (2006). Decision Making with The Analytic Networking Process; Economic, Political, Social and Technological Applications with Benefits, Opportunities, Costs and Risks. Springer.

Yoon Been Lee \& JiYoung Park. (2011). Assessment system for feasibility analysis of national R\&D programs: The case of Korea. International Journal of Innovation and Technology Management, 8, 661-676

Sang-Jin Ahn and Yoon Been Lee. (2013). Ex ante Evaluation Framework for R\&D Program: Exercises from Korea Government. International Journal of Innovation, Management and Technology, 4, 117-121. 